\title{
Two Consecutive Successful Pregnancy Outcomes in a woman with Eisenmenger's Syndrome: A Case Report
}

\author{
Nooshin Amjadi' ${ }^{1}$, Maryamsadat Hosseini' ${ }^{2}$, Soha Mirreza ${ }^{3 *}$ and Forough Mortazavi ${ }^{4}$ \\ ${ }^{1}$ Gynecologist, Assistant Professor, Department of Obstetrics and Gynecology, Preventative Gynecology Research Center (PGRC), Imam \\ Hossein Hospital, Shahid Beheshti University of Medical Sciences, Tehran, Iran
}

${ }^{2}$ Gynecologist, Associate professor, Department of Obstetrics \& Gynecology, Faculty of Medicine, Shahid Beheshti University of Medical Sciences, Tehran, Iran

${ }^{3}$ Resident of Gynecology, Department of Obstetrics and Gynecology, Preventative Gynecology Research Center (PGRC), Imam Hossein Hospital, Shahid Beheshti University of Medical Sciences, Tehran, Iran

${ }^{4}$ Associate professor, Department of Midwifery, School of Medicine, Sabzevar University of Medical Sciences, Sabzevar, Iran

*Corresponding author: Soha Mirreza, Department of Obstetrics and Gynecology, Preventative Gynecology Research Center (PGRC), Imam Hossein Hospital, Imam Hossein square, Shahid Beheshti University of Medical Sciences, Tehran, Iran

\begin{abstract}
Eisenmenger's syndrome is a very rare condition in pregnant women. The incidence of ES is about 3\% of the pregnant patients with congenital heart defects; however, it can be accompanied with high incidence of maternal and neonatal morbidity and mortality. Therefore, these patients should have efficient contraception or termination of pregnancy in the first trimester. We present two poorly-controlled consecutive pregnancies with good outcomes in a woman with ES to clarify the appropriate function of teamwork in the management of emergency situations in similar cases. Alongside teamwork, good prenatal care is also important because it can result in elective termination in higher gestational age of pregnancy.

A 21-year old woman, Repeat II cesarean belonging to a very low socioeconomic class, with ES was admitted to the emergency ward of Imam Hossein teaching hospital affiliated to Shahid Beheshti University of Medical Sciences with labor pain and severe dyspnea in 28 weeks of pregnancy. Echocardiography indicated a PAP of $120 \mathrm{mmHg}$. The patient had supportive treatment in intensive care unit until she was discharged. Despite previous reports of poor pregnancy outcomes in women with ES, high quality and significant treatment through labor and postpartum period lead to good outcomes in both mother and neonate.
\end{abstract}

Keywords: Eisenmenger complex; outcome; pregnancy; pulmonary hypertension

\section{Introduction}

Several congenital heart defects may result in Eisenmenger's syndrome (ES) [1]. The progress of ES in patients with congenital heart defects depends on the heart defect size and location [2]. Signs and symptoms of ES include right ventricular (RV) failure due to right ventricular hypertrophy, nail clubbing, cyanosis, dyspnea, edema, fatigue, dizziness, and arrhythmia [1]. For the first time in 1897, Victor Eisenmenger described a 23-year-old man with a large ventricular septal defect and pulmonary arterial hypertension and termed the condition as ES [3]. The major causes of death in
ES are right ventricular failure, pulmonary hypertension crisis, arrhythmia and stroke [1]. ES is a very rare condition in pregnant women. The incidence of ES is about 3\% of the pregnant patients with congenital heart defects [4]. Although ES progresses slowly in non-pregnant women, the increased blood volume during pregnancy may advance the disease during a relatively short time [5]. When pulmonary hypertension exceeds $70 \%$ of systemic blood pressure, pregnancy may be associated with complications and cause maternal death [6]. In a review, the maternal mortality rate from 1978 through 1996 due to ES was 36\% and the risk of 
maternal death remained unchanged over the period [5]. Neonatal outcome of pregnancy with ES is also poor. ES is a strong risk factor for spontaneous abortion, preterm birth, and intrauterine growth retardation (IUGR) [7].

As a rule, pregnancy in women with ES must be prevented or terminated in the first trimester $[1,4,8,9]$. In women who choose to continue pregnancy, a team consists of an obstetrician, perinatologist, cardiologist and an anesthesiologist is needed to care the pregnancy and labor [4,9]. Women with ES should be hospitalized in the second trimester of pregnancy [10]. Pulmonary vasodilator agonists have been used for lowering pulmonary hypertension with good results $[9,11,12]$. The best mode of delivery is a non-instrumental vaginal delivery using a labor pain relief through epidural block. Spinal analgesia is also preferred for cesarean in these women [5,9]. In general, treatment of ES is supportive [13] and includes oxygen therapy, the use of digitalis, diuretics, vasodilators and anticoagulants [4]. Usually anticoagulant therapy is prescribed to prevent thrombotic events; however, it may increase the risk of hemorrhage in the postpartum. Kahn reported a pregnancy in a 23-year old woman G4P3 with ES who was transferred to the hospital at 38 weeks with a two-month history of dyspnea and edema. After vaginal birth, she was treated with heparin to prevent thromboembolism. In the next day, severe vaginal bleeding developed and finally she expired [8]. We present two consecutive pregnancies with ES and good outcomes in a 21year old woman, with poor prenatal care. If she had regular visits during her pregnancy by specialists in obstetric, perinatology and cardiology she would have a higher chance of elective termination in later gestational age of her pregnancies; however, in emergent situations, a good teamwork can lead to saving both the patient and her neonate as it happened in both pregnancies in this patient. Therefore, women with ES may have a chance to experience motherhood.

\section{Case Presentation}

A 21-year old mother G1P0 in 34 weeks of pregnancy was admitted in the emergency ward of Imam Hossein teaching hospital affiliated to Shahid Beheshti University of Medical Sciences with labor pain and dyspnea. She was a known case of ES since 25 weeks of her pregnancy. The most important findings were pulse rate (PR) of $100 \mathrm{beat} / \mathrm{min}$, respiratory rate (RR) of $24 / \mathrm{min}$, blood pressure (BP) of $100 / 60 \mathrm{mmHg}$, 02 SAT of $92 \%$ and no cyanosis was seen. The echocardiography revealed a mild right ventricular enlargement, a mild left atrial enlargement, a mild reduced right ventricular function, a mild left ventricular hypertrophy (LVH), ejection fraction (EF) of 50\%, Pulmonary artery systolic pressure (PAPs) of $50 \mathrm{mmHg}$, and a large ventricular septal defect (VSD) progressing to ES. She continued her pregnancy until presented dyspnea and cyanosis in the 34th week of pregnancy. The vital signs were as follows: $P R=100$ beat $/ \mathrm{min}, \mathrm{RR}=34 / \mathrm{min}$ and $\mathrm{BP}=100 / 70 \mathrm{mmHg}$. The second echocardiography reported EF of $50 \%$ and PAP of 98
mmHg. $\mathrm{O}_{2}$ SAT was $86 \%$. Due to severe pulmonary hypertension, viability of fetus and low Bishop score, cesarean was performed, and a neonate was born with Apgar score 9/10 and birth weight of $2 \mathrm{~kg}$. She was admitted to the intensive care unit (ICU) and after four days, she was discharged from the hospital in good condition.

Again, in her second pregnancy, she was admitted for an elective therapeutic abortion in the $16^{\text {th }}$ week of pregnancy but she did not accept and left the hospital. In physical examination she had PR of 110 beat/min, RR of $32 / \mathrm{min}$, BP of $110 / 70 \mathrm{mmHg}$ and $\mathrm{O}_{2}$ SAT of $85 \%$. The patient also presented acrocyanosis. Echocardiography in the $16^{\text {th }}$ week of pregnancy detected EF of $50 \%$, PAP of $80 \mathrm{mmHg}$, mild systolic dysfunction and mild right ventricle enlargement. In electrocardiography, a sinus tachycardia was seen. After 12 weeks, she hospitalized in emergency ward with labor pain and dyspnea. Blood pressure, pulse rate, and respiratory rate were 100/70 mm Hg, 112 beat/min, and 30/min, respectively. Finding in arterial blood gas analysis (ABG) were as follows: $\mathrm{PH}=7 / 52$, partial pressure of carbon dioxide $\left(\mathrm{PCO}_{2}\right)=18 \mathrm{~mm} \mathrm{Hg}$, bicarbonate $\left(\mathrm{HCO}_{3}\right)=27 \mathrm{mEq} \backslash \mathrm{L}$ and $02 \mathrm{SAT}=90 \%$. In the electrocardiogram, a sinus tachycardia was detected. Echocardiography indicated a PAP of $120 \mathrm{mmHg}$. Immediately oxygen was administered, emergency consultation with a cardiologist was done According to consultation with an anesthesiologist, pethidine was injected to reduce the labor pain. Supportive management such as oxygen and pain control continued. While stabilizing the patient, magnesium sulfate for neuroprophylaxis of the baby and betamethasone for fetal lung maturity were administered. The patient was admitted to the ICU. After 8 hours, because of intensified labor pain, cesarean was done under general anesthesia and a preterm baby girl with Apgar score of $7 / 8$ and birth weight of $1800 \mathrm{gm}$ was born. Tubal ligation was performed and again she was transferred to the ICU ward. Enoxaparin was administered to prevent thromboembolic events in the postpartum period. The result of the echocardiography after cesarean showed PAPs of $110 \mathrm{~mm} \mathrm{Hg}$ and EF of $48 \%$. Four days later, she transferred to the post-cesarean ward and after one week, she discharged with good general condition.

\section{Ethics}

We obtained patient's informed consent for publishing this report.

\section{Discussion}

It is strongly recommended that women with ES be discouraged having pregnancy or be advised to terminate in the first trimester of pregnancy [4,9]; however, few reviews and case reports indicated that outcomes for women with ES have been improved [11-16]. Geohas and McLaughlin reported a 21-year old woman G3P2 in the $34^{\text {th }}$ week of pregnancy with ES who suffered from dyspnea and edema in the third trimester. She was treated with epoprostenol and was terminated by cesarean. The outcome was good and a newborn with good Apgar score was born [13]. Our patient was belonging 
to an underprivileged social group and did not have appropriate prenatal care during her pregnancies. She was a known case of ES since second trimester of her first pregnancy which was continued until gestational age of 34 weeks. In her second pregnancy, she maintained her pregnancy and finally was terminated in 28 weeks of pregnancy. The outcome of both pregnancies was good similar to the results reported in previous studies $[10,13,17]$ while in Duan's report, the perinatal outcome of pregnant women with ES were poor [1]. In a systematic review from 1978 through 1996 on 73 women with ES, patient`s age was a risk factor of maternal death [5]. Our patient was young, and it can be one of the reasons leading to good outcome. In addition, $\mathrm{O} 2$ sat and hemoglobin did not show any relationship to the outcome in ES [5].

Although vaginal delivery is preferred in these patients [4] other factors such as Bishop score and maternal and fetal condition are important to determine delivery route. In our case cesarean was preferred. In three reviews, $65 \%$ to $100 \%$ of patients gave birth by cesarean due to deteriorating maternal condition during the third trimester of pregnancy $[4,12,18]$. In a review study by Wang, and colleagues on 13 pregnancies, no pregnancy continued to term [19]. In our study, both pregnancies were preterm and terminated at the gestational age of 34 and 28 weeks of pregnancy, respectively. Despite poorly controlled prenatal care, the mother saved because she was young and received proper care in a tertiary center during labor and postpartum period. Besides, preterm labor at the $34^{\text {th }}$ and $28^{\text {th }}$ week of pregnancy may contribute to the successful outcomes of the patient due to stopping the progress of hemodynamic changes and worsening the condition. On the other hand, the patient was referred to the same hospital and we had access to her past medical history. Because she was not supervised between her pregnancies, we were not aware of the medications and the cares she received and whether she was adherent to the treatment.

\section{Conclusion}

Although prevention of pregnancy or termination in the first trimester is usually recommended in patients with ES, in this case, two consecutive pregnancies developed with good outcomes. Although the patient received no proper prenatal care including visiting a cardiologist before pregnancy and having timely care by a perinatologist or even an expert obstetrician in managing high risk patients, receiving a significant treatment in a tertiary center during labor and postpartum period resulted in good outcomes in this patient. Women with ES may have a chance to experience motherhood.

\section{Acknowledgment}

Authors thank the patient for agreeing to publish this report.

\section{Ethical Approval}

We obtained patient consent for publishing this report.

\section{References}

1. Duan R, Xu X, Wang X, Yu H, You Y, et al. (2016) Pregnancy outcome in women with Eisenmenger's syndrome: a case series from west China. BMC Pregnancy Childbirth 16: 356.

2. Beghetti M, Galie N (2009) Eisenmenger syndrome a clinical perspective in a new therapeutic era of pulmonary arterial hypertension. J Am Coll Cardiol 53: 733-740.

3. Daliento L, Somerville J, Presbitero P, Menti L, Brach-Prever S, et al. (1998) Eisenmenger syndrome. Factors relating to deterioration and death. Eur Heart J 19: 1845-1855.

4. Yuan SM (2016) Eisenmenger Syndrome in Pregnancy. Braz J Cardiovasc Surg 31: 325-329.

5. Weiss BM, Zemp L, Seifert B, Hess OM (1998) Outcome of pulmonary vascular disease in pregnancy: a systematic overview from 1978 through 1996. J Am Coll Cardiol 31: 1650-1657.

6. Siu SC, Sermer M, Colman JM, Alvarez AN, Mercier LA, et al. (2001) Prospective multicenter study of pregnancy outcomes in women with heart disease. Circulation 104: 515-521.

7. Abbas AE, Lester SJ, Connolly H (2005) Pregnancy and the cardiovascular system. Int J Cardiol 98: 179-189.

8. Kahn ML (1993) Eisenmenger's syndrome in pregnancy. N Engl J Med 329: 887 .

9. Niwa K. Adult Congenital Heart Disease with Pregnancy. Korean Circ J. 2018;48: 251-276.

10. Mukhopadhyav P, Bhattacharya P, Begum N (2012) Successful Pregnancy Outcome with Eisenmenger Syndrome. Journal of Obstetrics and Gynaecology of India 62: 68-69.

11. Bedard E, Dimopoulos K, Gatzoulis MA (2009) Has there been any progress made on pregnancy outcomes among women with pulmonary arterial hypertension? Eur Heart J 30: 256-265.

12. Curry RA, Fletcher C, Gelson E, Gatzoulis MA, Woolnough M, et al. (2012) Pulmonary hypertension and pregnancy--a review of 12 pregnancies in nine women. BJOG 119: 752-761.

13. Geohas C, McLaughlin VV (2003) Successful management of pregnancy in a patient with eisenmenger syndrome with epoprostenol. Chest 124 : 1170-1173.

14. Wang H, Liu T (2013) Pregnancy and hemolysis, elevated liver enzymes and low platelet count syndrome in patients with Eisenmenger's syndrome. Am J Med Sci 346: 385-389.

15. Tokgoz HC, Kaymaz C, Poci N, Akbal OY, Ozturk S (2017) A successful cesarean delivery without fetal or maternal morbidity in an Eisenmenger patient with cor triatriatum sinistrum, double-orifice mitral valve, large ventricular septal defect, and single ventricle who was under long-term bosentan treatment. Turk Kardiyol Dern Ars 45: 184-188.

16. Rathod S, Samal SK (2014) Successful pregnancy outcome in a case of eisenmenger syndrome: a rare case report. J Clin Diagn Res 8: OD08OD09.

17. Guo XY, Shao H, Chen YS, Liu CY, Zhang Y, et al. (2014) [Eisenmenger's syndrome in pregnancy: a case report and literature review]. Beijing Da Xue Xue Bao Yi Xue Ban 46: 986-989.

18. Avila WS, Grinberg M, Snitcowsky R, Faccioli R, Da Luz PL, et al. (1995) Maternal and fetal outcome in pregnant women with Eisenmenger's syndrome. Eur Heart J 16: 460-464.

19. Wang H, Zhang W, Liu T (2011) Experience of managing pregnant women with Eisenmenger's syndrome: maternal and fetal outcome in 13 cases. J Obstet Gynaecol Res 37(1): 64-70. 


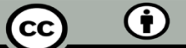

This work is licensed under Creative

Commons Attribution 4.0 License

To Submit Your Article Click Here: Submit Article

DOI: $10.32474 /$ IGWHC.2019.03.000156

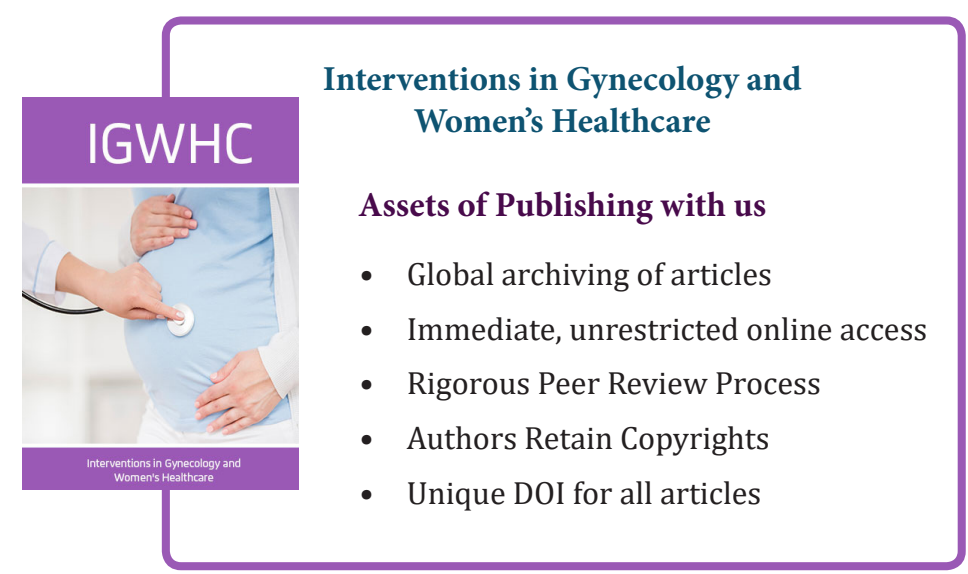

